\title{
Trabalhonecessário
}

V.16 no 31 / 2018 ISSN: 1808-799 X

\author{
Universidade Federal Fluminense \\ Faculdade de Educação
}

\section{NEDDATE - NÚCLEO DE ESTUDOS, DOCUMENTAÇÃO E DADOS SOBRE TRABALHO E EDUCAÇÃO REVISTA TRABALHO NECESSÁRIO: http://periodicos.uff.br/trabalhonecessario}

Redação: R. Professor Waldemar Freitas Reis, s/nº, bloco D, sala 525, Gragoatá - São Domingos, Niterói RJ, CEP 24210-201 - revistatrabalhonecessario@gmail.com

\section{EDITORES}

Lia Tiriba, Maria Cristina Paulo Rodrigues e José Luiz Cordeiro Antunes

\section{CONSELHO EDITORIAL}

Caridad Perez García (UCPEJV - Cuba),Celso Ferretti (UNISO - Brasil), Gaudêncio Frigotto (UFF/UERJ Brasil), José Claudinei Lombardi (UNICAMP - Brasil), Maria Ciavatta (UFF - Brasil), Roberto Leher (UFRJ Brasil), Tomás Rodrigues Villasante (UCM - Espanha), Sonia Maria Rummert (UFF) eVirgínia Fontes (UFF/EPJV / Fiocruz - Brasil).

\section{COMITÊ CIENTÍFICO}

Alexandre Maia do Bomfim (IFRJ), Ana Margarida Campello (EPSJV/FIOCRUZ), Ana Motta (UFF), André Feitosa (EPSJV/FIOCRUZ), André Martins (UFJF), Andrea Araújo Vale (UFF), Anita Handfas (UFRJ), Angela Siqueira (UFF), Angela Tamberlini (UFF), Aparecida Tiradentes (EPSJV/FIOCRUZ, in memoriam), Claudio Fernandes da Costa (UFF),Célia Regina Vendramini (UFSC),Daniela Motta (UFJF),Dante Moura (IFRN), Deise Mancebo (UERJ),Domingos Leite Lima Filho (UTFPR),Dora Henrique da Costa (UFF), Edison Oyama (UFRR),Edson Caetano (UFMT),Eneida Oto Shiroma (UFSC), Eraldo Leme Batista (UNIVAS-MG),Eunice Trein (UFF),Eveline Algebaile (UERJ),Filippina Chinelli (EPSJV/FIOCRUZ),Flávio Anício (UFRRJ),Francisco José Lobo Neto (FIOCRUZ), Guadelupe Teresinha Bertussi (UNAM e UFSC), Hajime Nozaki (UFMS e UFJF), Henrique Tahan Novaes, Ivo Tonet (UFAL), Jacqueline Botelho (UFF),Jaqueline Ventura (UFF), João dos Reis da Silva Jr. (UFSCar), José dos Santos Souza (UFRRJ),José Luiz Cordeiro Antunes (UFF),Júlio Cesar França Lima (FIOCRUZ),Justino de Souza Junior (UFC), Kátia Lima (UFF),Laura Souza Fonseca (UFRGS), Lea Calvão (UFF), Lia Tiriba (UFF),Lígia Klein (UFPR), Luciana Requião (UFF),Marcelo Lima (UFES), Maria Clara Bueno Fischer (UFRGS), Maria Cristina Paulo Rodrigues (UFF), Maria Inês do Rego Monteiro Bomfim (UFF), Maria de Fátima Félix Rosar (UNICAMP),Marcia Alvarenga (UERJ), Mariléia Maria da Silva (UDESC), Marisa Brandão (CEFET-RJ), Marise Ramos (UERJ,FIOCRUZ), Marlene Ribeiro (UFRGS), Myriam Feldfeber (UBA Argentina), Ney Luiz Teixeira Almeida (UERJ), Olinda Evangelista (UFSC), Ramon de Oliveira (UFPE), Raquel Varela (Universidade Nova de Lisboa - Portugal), Roberto Leher (UFRJ), Ronaldo Lima (UFPA), Rosilda Benacchio (UFF), Rui Canário (Universidade de Lisboa - Portugal), Sandra Maria Siqueira (UFBA), Sandra Morais (UNIRIO), Sérgio Lessa (UFAL), Sonia Maria Rummert (UFF), Susana Vasconcellos Jimenez (UFC), Tatiana Dahmer (UFF), Valdemar Sguissardi (UFSCar), Vania Motta (UFRJ) e Zuleide Silveira (UFF)

\section{ORGANIZAÇÃO DA TN 31 (2018)}

Profs. Drs. Doriedson do Socorro Rodrigues e Ronaldo Marcos de Lima Araújo - GEPTE / UFPA

\section{ASSISTENTES DE EDIÇÃO}

Brunna Santana Ribas, Daniel Tiriba, Jean Pablo Silva de Lima, Sandra Butschkau, Victor Hugo Raposo Ferreira, Victória Sias e William Kennedy do Amaral Souza

FOTO DA CAPA

Por do Sol no Pantanal Matogrossense-MT, 2016, autoria de Lia Tiriba

\section{MONTAGEM DA CAPA}

Daniel Tiriba 


\section{Trabalhonecessário}

V.16 no 31 / 2018 ISSN: 1808-799 X

Indexado por / Indexed by
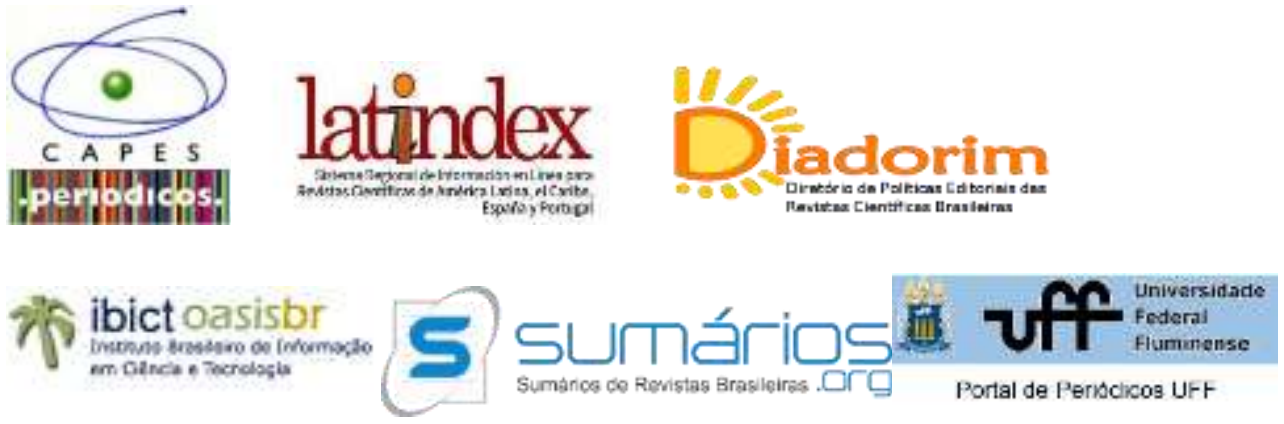

Apoio:

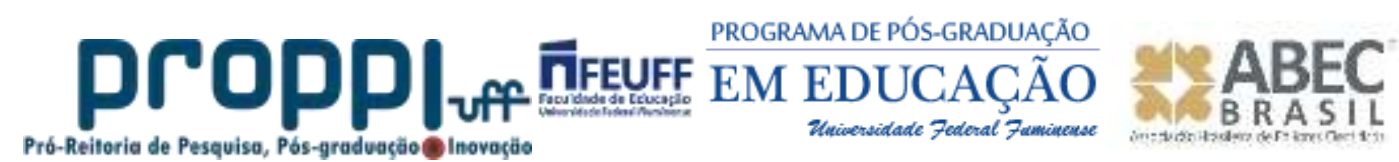

Ficha Catalográfica elaborada pela Biblioteca Central do Gragoatá/SDC/UFFBibliotecária:

Mahira de Souza Prado CRB-7/6146

\footnotetext{
- T758TrabalhoNecessário [recurso eletrônico]/Universidade Federal

Fluminense, Faculdade de Educação, Núcleo de Estudos, Documentação e Dados sobre Trabalho e Educação. - Ano 1, n. 1

(2003)-. - Niterói: NEDDATE, 2003-.
}

Anual, 2003-2007; Semestral, Jan./Jun. 2008-Jul./Dez. 2014;

Quadrimestral, Jan./Abr. 2015-.

Modo de acesso: World Wide Web.

Título extraído da home page (acesso em 06 mar. 2018).

Disponivel em: http://www.uff.br/trabalhonecessario/

ISSN: $1808-799 \mathrm{x}$

1. Educação. 2. Ensino. I. Universidade Federal Fluminense. Faculdade de Educação. II.

Universidade Federal Fluminense. Núcleo de Estudos, Documentação e Dados sobre Trabalho

e Educação. 